\title{
Self-configuration and self-optimization process with taguchi method in hybrid optical wireless network
}

\author{
Adam Wong Yoon Khang ${ }^{1}$, Arnidza Ramli ${ }^{2}$, Shamsul J. Elias ${ }^{3}$, J. Pusppanathan ${ }^{4}$, \\ Jamil Abedalrahim Jamil Alsayaydeh ${ }^{5}$, Fatin Hamimi Mustafa ${ }^{6}$, Win Adiyansyah Indra ${ }^{7}$, \\ Johar Akbar Mohamat Gani ${ }^{8}$ \\ ${ }^{1}$ Broadband \& Networking Research Group (BBNET), Center for Telecommunication Research and Innovation, \\ Fakulti Teknologi Kejuruteraan Elektrik and Elektronik (FTKEE), Malaysia \\ ${ }^{5,7}$ Center for Advanced Computing Technology, Fakulti Teknologi Kejuruteraan Elektrik and Elektronik (FTKEE), \\ UTEM, Malaysia \\ ${ }^{4}$ School of Biomedical Engineering \& Health Sciences, UTM, Malaysia \\ ${ }^{3}$ Department of Computer Technology and Networking, UITM, Malaysia \\ ${ }^{2}$ Photonic Technology Centre, Faculty of Electrical Engineering, Universiti Teknologi Malaysia, Malaysia \\ ${ }^{8}$ Fakulti Teknologi Kejuruteraan Elektrik dan Elektronik (FTKEE), UTEM, Malaysia \\ ${ }^{6}$ Institute for Research in Molecular Medicine (INFORMM), Universiti Sains Malaysia, Malaysia
}

\section{Article Info}

Article history:

Received Jan 29, 2020

Revised Mar 6, 2020

Accepted Mar 25, 2020

\section{Keywords:}

AODV-UU

ESCMDR

FiWi-MANET

PDR

PLP

\begin{abstract}
In this paper, an alternative improvement is proposed which is the adaptive wireless access networks-based optical backhaul convergence that will greatly promote to use the existing resource of MANET (mobile ad hoc network). However, these characteristics itself acts as a drawback to the MANET applications such as the random distribution of nodes and continuously changing topology. MiNiTab statistical software was used to model the effect of the parameter variation to predict the field quality through the design of experiments while OMNeT++ network simulation was created to visualize the effect of QoS performance study in response with varying speed scenario. The result shows that the proposed ESCMDR scheme can obtain robustness and outperformed compared to the nonTaguchi previous study when it is used in random waypoint mobility model in any speed of sources. The work is based on Packet Delivery Ratio (PDR) and Packet Loss Probability (PLP) metric under the varying speed scenario. It results in better QoS network PDR of $28.9 \%$ improvement, with $83.56 \%$ improvement on average PLP. The paper shows that the MANET QoS performance constrained can be addressed with the self-configured data rate of integrated optimization with Taguchi method on AODV-UU (Adhoc On Demand Distance Vector-Uppsala University) routing technique.
\end{abstract}

Copyright $\odot 2020$ Institute of Advanced Engineering and Science. All rights reserved.

\section{Corresponding Author:}

Adam Wong Yoon Khang,

Broadband \& Networking Research Group (BBNET),

Center for Telecommunication Research and Innovation,

Fakulti Teknologi Kejuruteraan Elektrik and Elektronik (FTKEE),

UTEM, Hang Tuah Jaya, Melaka, Malaysia.

Email: adamwong@utem.edu.my

\section{INTRODUCTION}

The mobility of wireless nodes in MANET can be regarded as time-variant at all time which varies dynamically within the network [1-3]. Hence, the identified link failure and QoS are considered as an open challenge in the field of MANETs. MANET consists of mobile peers where information is transferred could be used for multiple networking technologies such as the IEEE 802.11 or Zigbee in a multi-hop fashion to 
different network topologies and scenarios [4-6]. This could have called for robust adaptive in the design of protocols to the adhered multi-scenario phenomenon in MANET. This brings to realization of better performance of resource-efficient and reliable wireless mobile networks [7, 8] in an unpredictable network condition. From the green integrated design process standpoint, the work here performs to better and enhanced access network condition in the hope of sustaining and improving their network performance $[9,10]$. Based on this criteria, the author in [11] introduced the QoS framework for Wireless Ad hoc Networks. This framework can dynamically adapt the flows with a suitable forwarding path between the sender and the targeted destination when reacting to observed changes based on user-supplied adaptation policy. In a more similar study such as $[12,13]$, it is believed that they had measured the MANET behavior by having a testbed in Android terminals connected to a management server to perform real time performance, considering the different performance metrics. It is for monitoring the network topology and packet flow of the transmission and reception. However, they did not use any Design of Experiment (DoE) method although they did propose a benchmark for users with the unique virtual experiment which would help them to capture the network condition information. It also did not emphasize over the interaction between parameters as well as the effect on the network process performance characteristic in MANET. There are other works in past studies closely related to the work's studies. By using the Taguchi approach, the researcher investigates the Dynamic Source Routing (DSR) performance with regard to MANET conditions $[14,15]$. Nevertheless, DSR routing is not resource-efficient in terms of alleviating the network lifetime, where it consumes time and energy to process any control data it received [16, 17]. Even if it is not the intended recipient for integrated green design process, this routing is probably the most widely accepted strategy in most of the research efforts, which currently is not viable in the respective design. Hence, an extended network integrated scheme of FiWi (Fiber-Wireless) based on ESCMDR approach is proposed by considering the Taguchi optimization AODV-UU routing in MANET via the IEEE 802.11g Distributed Coordination Function (DCF) mode [18, 19]. The paper is organized as follows: the next part describes the proposed method, while section 3 presents the results and their discussion and the last section would be to conclude this work.

\section{RESEARCH METHOD}

In this section, the two main steps that involved in this research are outlined accordingly. Section A will highlight the simulation planning enabled by cross layer design for integrated MAC (medium access control)/routing solutions while section B gives an overview of the proposed algorithm of Taguchi-based DoE to increase the efficiency exploitation of the available resources. Both sections are towards the FiWiMANET conceptual scheme.

\subsection{OMNET++ modules implementation for DOE simulation planning}

The developed modules and their capabilities will be briefly discussed in this section, without many technical or coding details. The following subsections are organized from access network to aggregation network. The Access Point (AP) entity in Figure 1 represents the mobile user terminals in the system. Using this node with MANET routing module in order to set the AODV-UU routing procedures, which then enables the internet gateway support for data packets. They are routed through it over the hybrid PON in ad hoc manner to the destination node of the packets, where in this work, it represents the infrastructure of the backhaul Internet server. The Network Layers module models the network and lower layers. The Network Interface module simulates the actual network interfaces condition to include energy model and routing. This is done in the Routing Table module.

The ONU module in Figure 1 was also implemented in the simulator in the same trend as the OLT (optical line terminal) module. The most important modules designed are in the Hybrid PON/ONU (optical network unit) optical interface. In this case, the model is made more selfconfigurable to the channels, which is based on a simple ranging protocol in the ONU discovery. The idea behind it is the ONU interface must be on a specific time and for a specific period of time. They operate as a layer 2 switches that have gates to connect to the physical layer and to connect to the upper layer (relay units in switches). When a frame is received from the higher layers, it is the EtherFrame with mobile station module that acts as hosts. While for the inner part of the OLT entity of Figure 1 includes three sub-layers, each one providing different functionality. The first sub-layer is the wdm where these interfaces are associated with physical devices, implemented in WdmPhy class. It can be subclassed as WdmInputPhy and WdmOutputPhy that can be referred as modeling physical reception and transmission interfaces respectively. Followed by the second sub-layer, is the scheduler where it uses grants for downstream traffic as well as upstream traffic. The scheduler also schedules time framing of downstream Ethernet frames in the Virtual Output Queueing (VOQs) status at the time of the arrival of the report message. 
VOQ is used to separate and protect memory spaces among traffic flows. The third sub-layer is the relay unit where it switches frames among Ethernet source.

In this section also, an integrated framework design called the hybrid FiWi-MANET network was proposed based on the combination of optical, wireless and self-configured route segmentation for the extended proposed algorithm for intended work. It is a bi-direction communication system network with downstream (from OLT to ONU) and upstream (from mobile user to gateway/ONU) packet flows. They are coupled with the resource utilization distributed approach of optimized multiple data rate link adaptation with selfconfigured capability. In this paper, since this is a DoE simulation work, a total of 8 experiments are performed using the OMNeT++ simulation tools. A value of 3 for the Random Network Generator is used in this work to reasonably predict better instead of using only a single random chance to obtain accurate and the best parameters value for optimizing the tested variables. All the simulation scenario under this work was run for 250 seconds and the run time intervals was set to 50 simulated seconds' intervals in order to obtain steady output under dynamic network. Based on Table 1, it is targeted for an $8 \mathrm{~km} \times 6 \mathrm{~km}$ area of simulation while the test run recommended to be done for medium-sized mall or school based on 2 ONUs as described in $[20,21]$ with UDP (user datagram protocol) traffic.

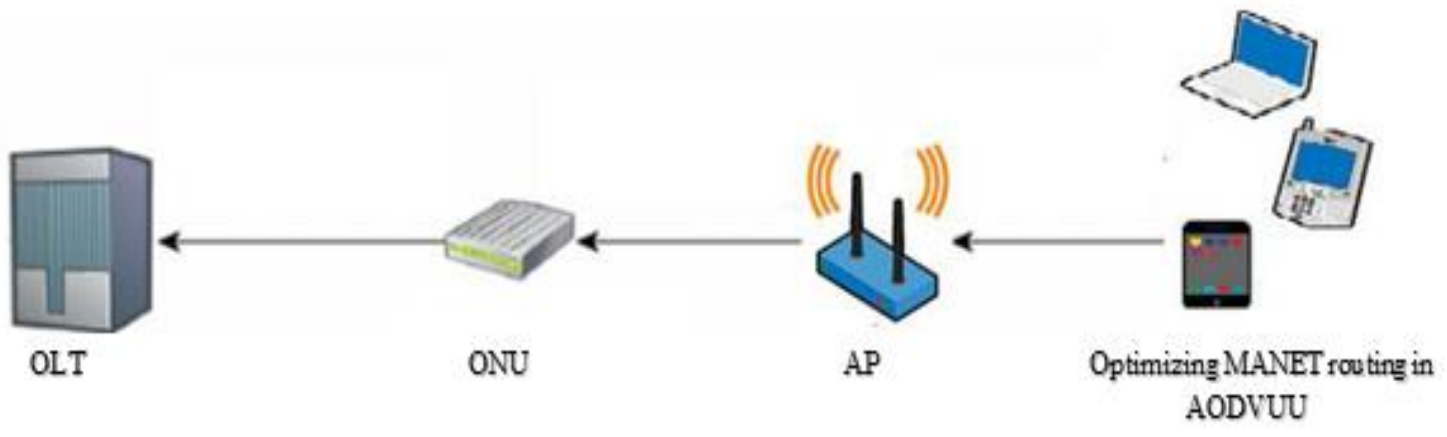

Figure 1. End-to-end traffic in hybrid FiWi-MANET framework architecture

Table 1. Fiber-Wireless-MANET common routing process parameter

\begin{tabular}{cc}
\hline Parameter & Values \\
\hline Fiber type & Single mode fiber \\
Reach (OLT-ONT) & $20 \mathrm{~km}$ \\
Number of OLT & 1 \\
Number of ONU & 2 \\
Number of nodes & 20 to 500 \\
Simulation Area & Max $8 \mathrm{~km} \mathrm{x} 6 \mathrm{~km}$ \\
Traffic type & UDP \\
Routing layer & AODV-UU \\
MAC layer & IEEE02.11g with DCF \\
Carrier frequency & $2.4 \mathrm{GHz}$ \\
Data rate & 6 Mbps, $24 \mathrm{Mbps}, 54 \mathrm{Mbps}$ \\
Message length (Packet size) & 512 byte, 1024 byte \\
Random number generator (RNG) & 3 \\
\hline
\end{tabular}

\subsection{Proposed algorithm}

This research is about the performance enhancements by parameterized approach to AODV-UU protocol of MANET and further connecting it with optical network for reliable transmission. From the study, there are a potential set of performance factors affecting the protocol capability to cope with the current network. Hence, Taguchi DoE was used to optimize the influence of the factors on the particular network performances [22]. Figure 2 presents the application of Taguchi method for optimal process setting of the simulated access network. Phase 1 and Phase 2 were the main key steps in order to look out the primarily process goal which is to establish a robust resource efficient QoS access network performance. By keeping the process goal relevant, it can ensure the existing objective setting to be selected for optimization that minimize the effects of the routing process variation. To achieve the robust and optimal conditions, there are a number of identified process parameters settings for tuning to be regarded as control factors such as the procedure of wait_on_reboot, checkNextHop, etc. These are illustrated also in Table 2. 


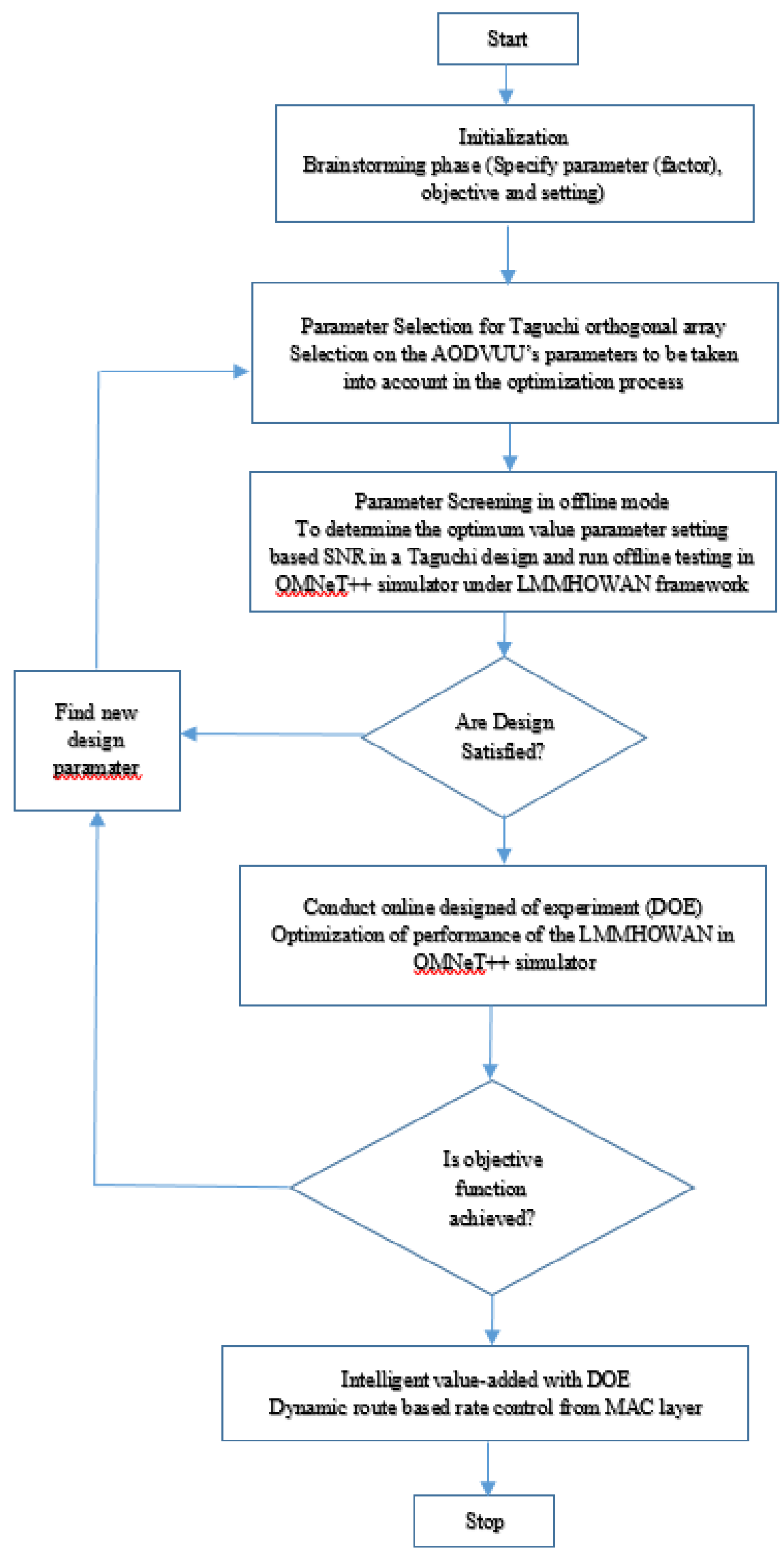

Phase 1

Phase 2

Phase 3

Phase 4

Figure 2. Systematic optimization processes in framework architecture 
Table 2. The optimal factors setting with respect to its value setting for orthogonal array design of Taguchi optimization

\begin{tabular}{ccccccccc}
\hline Factors & Level 1 & Level 2 & Level 3 & Level 4 & Level 5 & :Level 6 & Level 7 & Level 8 \\
\hline Rate limit (ms) & $1(-)$ & $1(-)$ & $1(-)$ & $1(-)$ & $10(+)$ & $10(+)$ & $10(+)$ & $10(+)$ \\
Wait_on_reboot ms) & $1(-)$ & $1(-)$ & $5(+)$ & $5(+)$ & $1(-)$ & $1(-)$ & $5(+)$ & $5(+)$ \\
Feedback (True/False) & $1(+)$ & $1(+)$ & $0(-)$ & $0(-)$ & $0(-)$ & $0(-)$ & $1(+)$ & $1(+)$ \\
Receive_n_hell os (ms) & $5(+)$ & $1(-)$ & $1(-)$ & $5(+)$ & $1(-)$ & $5(+)$ & $1(-)$ & $5(+)$ \\
Optimized_hell os (True/False) & $1(+)$ & $0(-)$ & $1(+)$ & $0(-)$ & $0(-)$ & $1(+)$ & $0(-)$ & $1(+)$ \\
checkNextHop (True/False) & $1(+)$ & $0(-)$ & $0(-)$ & $1(+)$ & $1(+)$ & $0(-)$ & $0(-)$ & $1(+)$ \\
\hline
\end{tabular}

From Table 2, it shows that the factors involved has a high (+) and low (-) level where each factor has two different levels and it can be seen as the proper array known as L8 $\left(2^{7}\right)$. It is due to its factor levels which are weighted equally across the entire design [23]. The optimal key factors are obtained from the parameter screening of Phase 3 in Figure 2, which are indicated by two types of Taguchi SN ratio performance characteristics, namely, "smaller-is-better" and "larger-is-better". Hence, the respective goal of the study is to increase the PDR and PLP performance criteria based on the optimal key factors found here. The key factors above are chosen using the engineering judgment such as the factor of wait_on_reboot can prevent a routing loop, which can cause unnecessary resource consumption such as packet loss probability [24]. Other than that, and in most cases of concerning mobile wireless users, they may experience undeliverable data packets and resource wastage due to redundancy transmission and dynamic transmission range when mobile end nodes are on the move. This scenario calls for optimization verification of the checkNextHop factor.

\section{RESULTS AND ANALYSIS}

In this section describes the simulation results and comparison between the proposed integrated MANET with green self-configure algorithm called the intelligence Last mile mobile hybrid optical wireless access network (inLMMHOWAN) and conventional MANET network which named as oRiG. To obtain the required result and analysis, the following metrics are used:

PLP [25, 26]: Packet delivery ratios also known as the probability of end-to-end delivery. The metric is considered as the node's estimate of the packet loss probability along the path between the node and the destination. The formula of packet loss probability along a link or path is defined as below:

1 minus the packet delivery probability along the corresponding link or path

where packet delivery probability $=\Sigma$ No of packets delivered $/ \Sigma$ No of packets send or created

Packet Delivery Ratio (PDR) [27]: The performance of throughput is measured through the PDR performance. This performance metric is used to determine the efficiency and accuracy of this framework paradigm. It is the ratio of the data packets received at the destinations to those generated by the sources. Higher packet delivery fraction indicates the better efficiency of the proposed improvement of optimization.

Packet delivery ratio $=\Sigma$ Number of packet received $/ \Sigma$ Number of packet send/created

In this section, 2 sets of parameter are presented which then are optimized in the form of output graph to be analyzed as follows:-.

\subsection{Packet delivery ratio (PDR)}

PDR is an important performance evaluation parameter for routing protocol to support network throughput. As referred to Figure 3, the increase of the node mobility speed causes the link break due to a collision during packet transmission which affects the reliability thus decreases the PDR values for mobile network performance of wireless domain. Every time a link is broken, a route error packet is generated and the varying mobility speeds of the mobile nodes influences the dependability of some route error packets. The route error packets, which are increasing and substantially transmitted at higher speeds, also cause data losses which are highly and likely to happen. To avoid that from happening, all transmission in the route discovery phase of the proposed scheme adopts the multiple routes based on multi-data rates, This to ensure that it is compliant with the optimum traffic profile despite the different speeds for quantifying the performance degradation in MANET, as well as to build a QoS route for packet forwarding in the source. Indirectly, routing loop can also be avoided which can consume more energy by the checkNextHop control factor that can subsequently increase the packet forwarding to the destination. This effect can promote the energy efficient consumption in this case. 


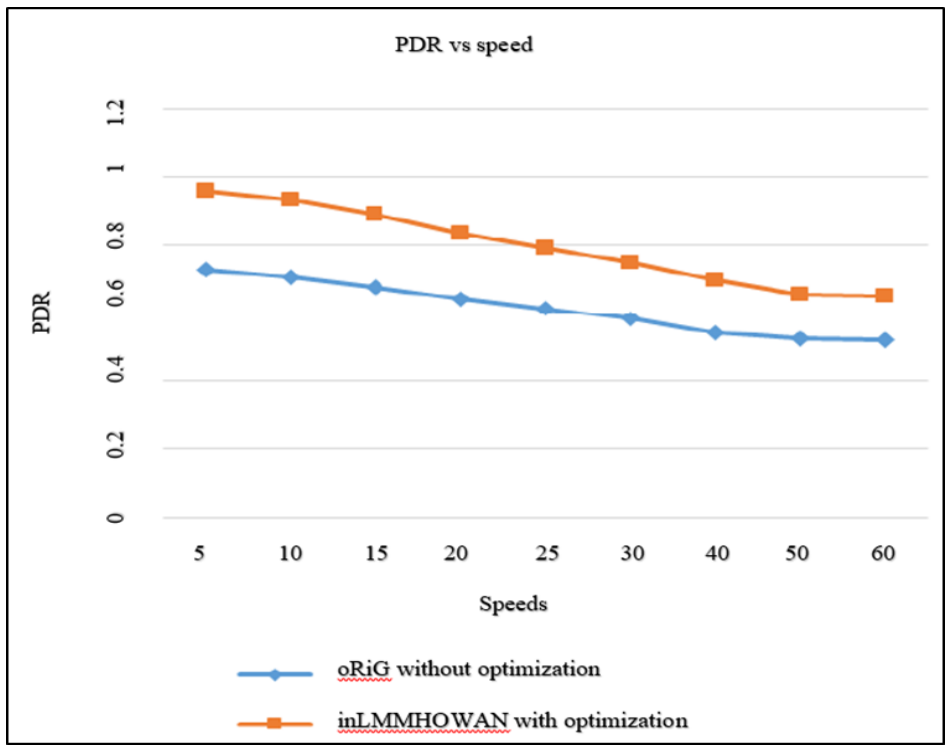

Figure 3. Performance of PDR for scenario A; oRiG and inLMMHOWAN multiparameter AODVUU routing before and after with self-configured mechanism based on taguchi optimization

\subsection{Packet loss probability (PLP)}

The network assessment continues with the packet loss probability as shown in Figure 4. This condition can be explained where the intermediate nodes are changing rapidly due to the increase of inherent factor user mobility rate. However, these results present the significant capacity improvements across all variation (low to high) of mobility usage scenarios subjected to minimize packet loss improvement; in this case, especially under low mobility, it can achieve about $83.56 \%$ average improvement at speed of $5 \mathrm{mps}$ compared to the oRiG parameter setting. This could be explained by good routing variables that can acquire paths with improved PLP in a multi-data rate wireless MANET compared to any routing variables in a single-rate wireless MANET. For example, by selecting the rate limit routing variable, it would place a limitation on the broadcast control messages such as route error messages (RERR) or HELLO message flooding in the network. Hence, the probability of choosing the stale route which may contribute to less packet delivery in the network can be lessened, which then can reduce the effect of packet loss.

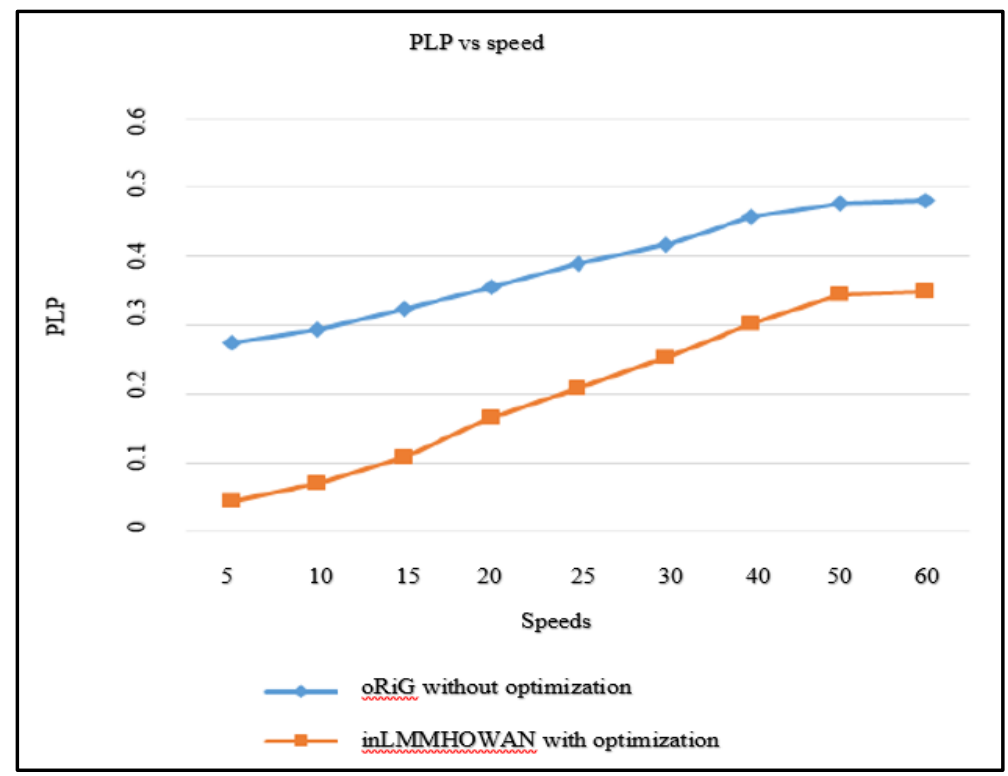

Figure 4. Performance of PLP for scenario A oRiG and inLMMHOWAN multiparameter AODVUU routing before and after with self-configure mechanism based on taguchi optimization 


\section{CONCLUSION}

The technologies that have been covered in this work are the major players as the last mile alternative given to the scenario of rapid growth in the field of mobile Internet access and mobile devices. Henceforth, in this article, by using the AODV-UU approach with appropriate parameters, it can reduce variation while provide increased performance in QoS efficiency. The choice of the optimum design parameters is based on the objective of performance study such as reducing the PLP or increasing the PDR criteria. Secondly, the work applies the self-configured of the multiple source data-rate through cross layer scheme from MAC layer to network layer to achieve the better QoS quality during the initial packet follow. The performance result of this proposed work is better QoS of resource consumption through information exchange across different layers, as well as its capability to combine functionality from different domains into one single functional entity. In conclusion, the proposed ESCMDR scheme under inLMMHOWAN framework of routing improvement work when compared to previous study of oRiq scheme achieved the average improvement of $28.9 \%$ in PDR and $83.56 \%$ in PLP respectively under the scenario of varying mobility nodes speed. For the future work, the work can have link layer improvement with IEEE 802.11n DCF MAC enhancement based on multi-antenna scheme (Multiple Input Multiple Output). It also can go beyond simulations and provide measurement results from a practical implementation.

\section{ACKNOWLEDGEMENTS}

The authors would like to express their deepest gratitude to Centre for Research and Innovation Management of Universiti Teknikal Malaysia Melaka (UTeM) under the Grant PJP/2019/FTKEE (5A)/S01658.

\section{REFERENCES}

[1] J. Manoranjini, et al., "Improved QoS and avoidance of black hole attacks in MANET using trust detection framework," Automatika:- Journal for Control, Measurement, Electronics, Computing and Communications, Jun pp. 1-11. 2019.

[2] Matheus, et al. "DYRP-VLC: A dynamic routing protocol for Wireless Ad-Hoc Visible Light Communication Networks," Ad Hoc Networks vol. 94, pp. 1-13, Nov 2019.

[3] P. H. Rantelinggi, et al., "Performance of Routing Protocol in MANET with Combined Scalable Video Coding, " Proc. EECSI 2017, Yogyakarta, Indonesia, pp. 19-21 September 2017.

[4] P. Bellavista, et al., "A Middleware Solution for Wireless IoT Applications in Sparse Smart Cities," Automatika: Journal for Sensors (Basel, Switzerland), vol. 17, pp. 1-18, Nov 2017.

[5] Bilbao J, Bravo E, Varela C, et al., "Developing the IoT through wireless communication networks: analysis of topologies, " Int J Biosen Bioelectron, vol.3, pp. 327-331, Nov 2017.

[6] Huan Hui Yan, et al., "Design and Development of Gas Leakage Monitoring System," Proceeding of International Conference on Electrical Engineering, Computer Science and Informatics (EECSI 2014), Yogyakarta, Indonesia, pp. 20-21 August 2014.

[7] Mahfuzur Rahman, et al., "Efficient Content Delivery for Mobile Communications in Converged Networks," Wireless Communications and Mobile Computing, vol. 2019, pp. 1-12, 2019.

[8] Cen, Nan, et al., "LANET: Visible-light ad hoc networks," Ad Hoc Networks, vol.84, pp. 107-123, March 2019.

[9] W. Hua-Hung, et al., " Effects of Green Innovation on Environmental and Corporate Performance: A Stakeholder Perspective," MDPI: - Journal for Sustainability, vol. 7, pp. 4997-5026, April 2015.

[10] M. A. Wong, et al., "Efficient P2P data dissemination in integrated optical and wireless networks with Taguchi method," TELKOMNIKA, vol. 17, pp 1642-1647. Aug 2019.

[11] A. Arjunan, et al., "Hyper-Erlang channel allocation factor-based QoS enhancement mechanism for mobile ad hoc networks," Elsevier Journal: - Alexandria Engineering, vol. 57, pp. 799-811, June 2018.

[12] Y. Tsutsui, et al., "Design of a Realtime Network Topology and Packet Flow Visualizing System for MANET Field Experiments," Second International IEEE Symposium on Computing and Networking Design, 2015, pp. 296-299.

[13] Naseeruddin, et al., "Application-Energy Prioritized Routing Algorithm for Wireless MANETs and its Experimental Evaluation," International Journal of Engineering and Advanced Technology (IJEAT), vol8, pp 664-670, June 2019.

[14] A.W.Y. Khang, et al., "Last mile mobile hybrid optical wireless access network routing enhancement," Bulletin of Electrical Engineering and Informatics, vol. 8, pp. 188-195, March 2019.

[15] K. Sahithi, Dr.S.P.Setty "Taguchi Design of Experiments for optimizing the Performance of AODV Routing Protocol in MANETS" International Journal of Engineering Research \& Technology, Vol. 7 Issue 04, April-2018 pp 519-526.

[16] Dr. Sunil Taneja, "Analysis of Packets Broadcast over MANET Protocols using UDP Traffic Pattern," International Journal of Engineering Sciences \& Research Technology, vol 3, pp. 547-553, February 2014.

[17] H Mohamed, et al., "Grey-based Taguchi Method in Multi-objective Optimisation of the Routing Process in Mobile Ad-hoc Networks," Journal of Applied Sciences, vol. 14, pp. 396-400, 2014. 
[18] K. Sahithi and S.P.Setty, "Taguchi Design of Experiments for optimizing the Performance of AODV Routing Protocol in MANETS," International Journal of Engineering Research \& Technology (IJERT), vol. 7, pp. 519-526, 2018.

[19] Hassan, Wan Hafiza Wan, et al., "Enhancement techniques of IEEE 802.11 wireless local area network distributed coordination function: A review." ARPN Journal of Engineering and Applied Sciences, vol. 13, pp. 1053-1062, 2018.

[20] P. Chakraborty, et al., "Design of Passive Optical Network for Hospital Management," Asian Journal for Convergence in Technology (AJCT), vol. 4, pp. 1-7, 2018.

[21] R. Shantha, et al., "Design of Wireless Passive Optical Communication Network Based On Fusion of Fiber to the X Architecture," International Journal of Engineering Research in Electronics and Communication Engineering, vol 4, pp 26-31, December 2017.

[22] M.A. Wong, et al., "QoS Support with Taguchi Method in Simulation Modeling Hybrid Architecture of Optical and Multi - Hop Wireless Ad Hoc Networks" International Journal of Engineering \& Technology (UAE), vol. 7, pp. 28-31, 2018.

[23] M.H.M. Alias, et al., "Analysis on the factors and effects of engine tapping noise by Design of Experiment (DOE)" Journal of Mechanical Engineering, vol. 12, pp. 27-48, March 2016.

[24] S. Lee, et al., "Link weight assignment and loop-free routing table update for link state routing protocols in energyaware Internet," Elsevier: Journal of Future Generation Computer Systems, Feb 2012, pp. 437-445.

[25] Y. Keshtkarjahromi, et al., "Content-Aware Instantly Decodable Network Coding over Wireless Networks," Proc. of IEEE ICNC, 2015, pp. 803-809.

[26] Marjasz R, et al., "Simulation Analysis of Packet Delivery Probability in LoRa Networks," Computer Networks. CN 2019. Communications in Computer and Information Science, vol. 1039, pp 86-98, June 2019

[27] A Jain, et al., "Simulation and Performance Analysis of Throughput and Delay on Varying Time and Number of Nodes in MANET," International Journal of Recent Research and Review, vol. 7, pp. 113-117, June 2014.

\section{BIOGRAPHIES OF AUTHORS}
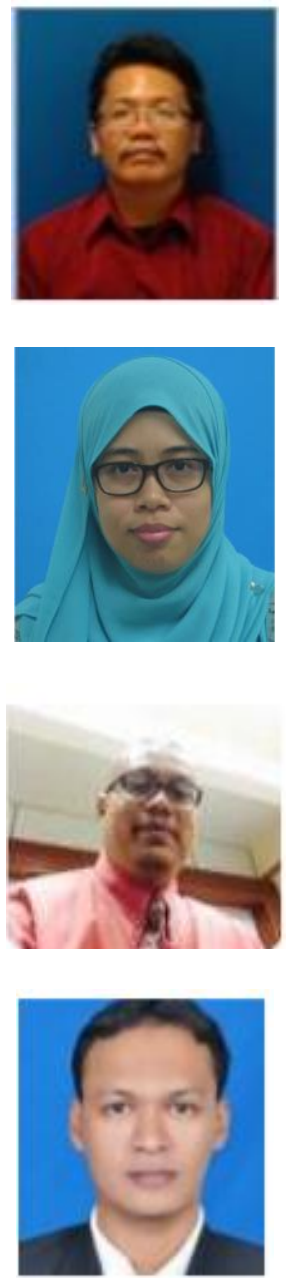

Adam Wong Yoon Khang received his Ph.D. Degree from Universiti Teknologi Malaysia in 2018. He is currently a Senior Lecturer in the Fakulti Teknologi Kejuruteraan Elektrik dan Elektronik, Universiti Teknikal Malaysia Melaka (UTeM). He is also the Professional Technologists for Malaysia Board of Technologists (MBOT). His current research interests are Internet of Things, Hybrid Optical Wireless, simulation optimization, ad hoc network and passive optical network but not limited to the mention topic here. He actively publishes research articles and received several research grants from the government and private sectors, university and international collaboration.

Arnidza Ramli received both the Bachelor degree in Electrical Telecommunication and Master degree in Electrical from the Universiti Teknologi Malaysia (UTM) in 2007 and 2009 respectively. She completed her $\mathrm{PhD}$ study in Electrical Engineering from the same university in 2017. She is currently a Senior Lecturer at School of Electrical Engineering, Faculty of Engineering, UTM and serve in Communication Engineering Division. Her current research activity is mainly focused on Energy efficiency for integrated optical and wireless network. Other research interests include optical communication and green networking.

Shamsul Jamel Elias completed his B. Computer Science from University of Oregon, USA. He received his Master degree in Business Administration from University of Hull at United Kingdom. He continues his PhD study in Computer Engineering at University Malaysia Perlis He then now a Senior Lecturer in University Teknologi Mara in year 2018 also as Senior Lecturer. His current research interest is VANETs, Congestion Control, Taguchi Method, Optimization, IoT but not limited to the mention topic here. Currently he supervised some postgraduate students as main and co-supervisor.

Jaysuman Bin Pusppanathan his Ph.D. degree from Universiti Teknologi Malaysia in 2016. He is currently a Senior Lecturer in the School of Biomedical Engineering \& Health Sciences, Faculty of Engineering, Universiti Teknologi Malaysia. His current research interests are electrical tomography for both process industry and biomedical applications, sensors and drone technology. He actively publishes research articles and receives several research grants from both the government and private sectors, university and international collaboration. 


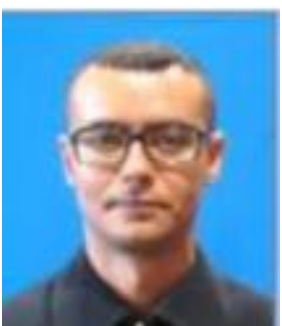

Jamil Abedalrahim Jamil Alsayaydeh received his Ph.D in National Mining University for Automation of Control Processes, Ukraine, in 2014. Then he joined University Teknikal Malaysia Melaka in year 2015 as Senior Lecturer at the Department of Electronics and Computer Engineering Technology in Technical University of Malaysia Malacca. His research interests include formal methods, simulation, Automatic Control of Process, computer system and networks, real time system and internet of things. He supervised 18 undergraduate students and is a reviewing member of various reputed journals.

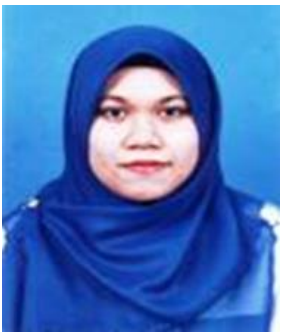

Fatin Hamimi Mustafa pursued $\mathrm{PhD}$ in Biomedical Engineering at University of Sydney, Australia and had successfully completed it in year 2016. Now, she joins Institute for Research in Molecular Medicine, Universiti Sains Malaysia, Kubang Kerian, Kelantan as Senior Lecturer position. Her current research interest are Internet of Things, Optical Biosensor, simulation optimization, and Optical Network. Currently she supervised two postgraduate students. One of her publications was published in Nature Publishing Group.

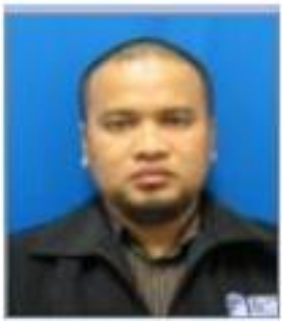

Win Adiyansyah Indra completed his B. Communication Engineering from Telkom University, Indonesia in year 2001. 10 years of working experience in Telco area, including Nokia. He received his Master degree from International Islamic University Malaysia, in year 2013, sponsored by Yayasan Khazanah Nasional. He then joined Universiti Teknikal Malaysia Melaka in year 2013 as Lecturer. His current research interest is Wireless Communication, Transmission, Radio Frequency Energy Harvesting.

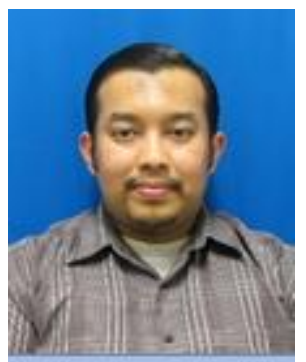

Johar Akbar bin Mohamat Gani completed his Bachelor of Engineering in Mechatronics with Honours from the International Islamic University Malaysia (IIUM) in year 2006. He worked as a layout engineer at the Penang Design Center, Intel Microelectronics (M) Sdn. Bhd. based in Penang and Kulim from 2007 till mid-2008. Later, He joined UTeM in April 2009 as a Teaching Engineer at the Faculty of Electrical and Electronic Engineering Technology (FTKEE), Universiti Teknikal Malaysia Melaka (UTeM). He is currently a research member of Centre for Robotics and Industrial Automation (CERIA). His research interest are Internet of Things, Electrical Energy Conversion and Human Assistive Machine. He is also became a member of Professional Technologists for Malaysia Board of Technologists (MBOT). 